\title{
Measuring the Performance of Parallel Information Processing in Solving Linear Equation Using Multiprocessor Supercomputer
}

\author{
Faten Hamad ${ }^{1} \&$ Abdelsalam Alawamrah $^{2}$ \\ ${ }^{1,2}$ Faculty of Educational Sciences, The University of Jordan, Amman, Jordan \\ Correspondence: Faten Hamad, Faculty of Educational Sciences, The University of Jordan, Amman, Jordan. E- \\ mail: F.hamad@ju.edu.jo
}

Received: May 21, 2017

doi:10.5539/mas.v12n3p74

\author{
Accepted: January 4, $2018 \quad$ Online Published: February 27, 2018 \\ URL: https://doi.org/10.5539/mas.v12n3p74
}

\begin{abstract}
Evaluating the performance of algorithms and its implementation methods play a major role in the assessment of the performance of many applications. It help researchers to decide which algorithm to use and which method to implement it. Furthermore, it give an indication of the performance of the hardware that the algorithm is tested over. In this paper we evaluate the performance of solving linear equation over supercomputer that consist of 64 processor running in parallel and using Message Passing interface (MPI) for information communication between processors. The sequential and multithreaded algorithm for solving linear equations has been experimented. The running time, speedup and efficiency of the algorithm has been calculated and the results showed that the parallel algorithm outperforms sequential and multithreaded methods when the matrix size is large $(8192 * 8192)$ and the number of processors is 64 . For large input data size, the results also showed that there is a noticeable decrease in running time as the number of processors increase. But in case of multithreaded the results showed that as the matrix size increase the time required for running the algorithm is rapidly increasing despite the increased number of threads.
\end{abstract}

Keywords: parallel computing, solving linear equation, multithreaded, super computer

\section{Introduction}

From the beginning of computer invention until today the computers processing has been developed very rapidly a new generation of computer systems sets higher standards with regards to performance, size, price, and usability. Meanwhile, the rapid developments which have occurred in computer hardware and software technology over the last two decades have made computers an essential and indispensable tool for different fields of our daily life( Alsayyed et al., 2017 ) (Hudaib et al,. 2007).

Computers continue to develop. In the 1990's, the Parallel Computers has been invented. Parallel computer systems adopt the idea of cooperation by employing multiple processors. The continued development of computer hardware and improvements in the computer price/performance ratio coupled with improvements in the usability and functionality parallel computers have raised the expectations of computers to the point where they appear to believe that any problem and any size model can be solved in short time, regardless of the size or complexity of the problem. Moreover, results are became very fast with parallel computers and they appear within hours instead of weeks or days. Thus, the race between the needs of the user and technological improvements continues to create a demand faster parallel computing (Alhadidi et al., 2006).

Large computational problems are divided, separately solved, and integrated into a final solution. Due to the advances in parallel computer architecture, machines with large numbers of processors are now available. Due to this technological progress, some researchers predict that "within a decade, all developments in computer applications and algorithm design will be taking place within the context of parallel computation" Parallel computation has motivated a considerable large number of researches due to advances in solid state, large scale integration of computer Performance, reliability and low cost of such digital devices as microprocessors which have led to the development of multi-processor computer architecture( Alhadidi et al.,2007). The concept of parallel processing is a departure from the trend of achieving increases in speed by performing single operations faster. Parallel processing achieves increases in speed by performing several operations in parallel

The high performance parallel computers have variety of hardware architectures that can be classified according 
to their way of manipulating the instructions and data. The most important high performance computer categories are as follows: SIMD machines: Single instruction machines that manipulate many data items in parallel. Such machines have large number of processors, ranging from 1,024 to 16,384. Vector processors are one type of SIMD machines. MIMD machines: These machines execute several instruction streams in parallel on different data. There are many kinds of MIMD systems that can be further classified according to their memory taxonomy as shared and distributed memory machines.

Parallel computers are used to solve large computational problems such as matrix multiplication. Matrix multiplication is a computer problem that has large input as many other numerical problems which require a large number of arithmetic operations, such computational problems require parallel computer to fast solve them. Also many applications to the sciences and engineering require the solution of very large in size linear systems of equations, and in many cases this task has been made feasible on modern computers. So it require super parallel computer to solve it.

Many researches has been done on parallel processing (Pasetto, and Akhriev, 2011, Maria, et al., 2015, Atif, and Rauf, 2009, Rajalakshmi, 2009, Delic, and Juric, 2013) many researches invistigate matrix and linear equation solving and numerical analysis over large number of processors using parallel processing (Scholl, Stumm and Wehn, 2013, Rajalakshmi, 2009, Saeed, et al., 2015), these researches focus on measuring the performance evolution of parallel processing and differentiate it from sequential analysis and parallelization of the sequential methods many researches discussed parallel algorithms based on Cholesky factorization, Gaussian elimination, LU decomposition, Gauss-Jordan, and many methods gave solution for dense linear systems,

Erich Kaltofen, and Victor Pan proposed Processor Efficient Parallel Solution of Linear Systems over an Abstract Field, The algorithms utilize within an $\mathrm{O}(\log \mathrm{n})$ factor as many processors as are needed to multiply two $\mathrm{n} \times \mathrm{n}$ matrices. Maria, et al., (2015) described a study of the Gaussian Elimination Applications, examine the utilizations of Gaussian Elimination technique. They showed that Progressive Gaussian Elimination technique is seen to be more quick, proficient and precise than that of Gaussian disposal strategy. The Gaussian Elimination technique is additionally proper for comprehending straight conditions on work associated processors. Delic, and Juric (2013), proposed a research that discuss some improvements of the Gaussian elimination method for solving simultaneous linear equations. Atif, and Rauf (2009) proposed an implementation of Gaussian elimination method to recover generator polynomials of convolution codes. Balasubramanya et al., (1994), proposed a new Gaussian elimination based algorithm for parallel solution of linear equations Scholl et al., (2013) proposed a Hardware implementations of Gaussian elimination over GF (2) for channel decoding algorithms.

This paper evaluate the performance of matrix multiplication and an application to it in solve linear equation on super computer, Gaussian elimination algorithm which is been used for solving a system of linear equations in parallel super computer.

\section{Overview of Solving Linear Equation Using Gaussian Elimination}

The goal of Gaussian elimination are to make the upper-left corner element a 1, use elementary row operations to get $0 \mathrm{~s}$ in all positions underneath that first 1 , get $1 \mathrm{~s}$ for leading coefficients in every row diagonally from the upperleft to lower-right corner, and get 0s beneath all leading coefficients. Basically, you eliminate all variables in the last row except for one, all variables except for two in the equation above that one, and so on and so forth to the top equation, which has all the variables. Then you can use back substitution to solve for one variable at a time by plugging the values you know into the equations from the bottom up. You accomplish this elimination by eliminating the $\mathrm{x}$ (or whatever variable comes first) in all equations except for the first one. Then eliminate the second variable in all equations except for the first two. This process continues, eliminating one more variable per line, until only one variable is left in the last line. Then solve for that variable.

The linear system problem is to find an $\mathrm{n}$-vector $\mathrm{x}$ such that $\mathrm{Bx}=\mathrm{S}$. Given an $\mathrm{n} \mathrm{x} \mathrm{n}$ nonsingular matrix $\mathrm{B}$ and an n-vector S.

The Solution of the linear systems $\mathrm{Bx}=\mathrm{S}$ is very significant and important in scientific and engineering computations. It is necessity for faster solutions in many areas of real-time computing, parallel algorithms, which promise to speedup computations. Programs using $\mathrm{p}$ processors should run $\mathrm{p}$ times faster than identical programs using only one processor, although a linear speed up might not be possible and the actual speed up is often much smaller.

A linear equations system of matrix $\mathrm{B}$ :

$\mathrm{B} 0,0 \times 0+\mathrm{B} 0,1 \times 1+\ldots+\mathrm{B} 0, \mathrm{n}-1 \mathrm{Xn}-1=\mathrm{S} 0$

$\mathrm{B} 1,0 \mathrm{X} 0+\mathrm{B} 1,1 \mathrm{X} 1+\ldots+\mathrm{B} 1, \mathrm{n}-1 \mathrm{Xn}-1=\mathrm{S} 1$ 


$$
\begin{aligned}
& \text { B } 2,0 \text { X } 0+B 2,1 X 1+\ldots \ldots+B 2, n-1 X n-1=S 1 \\
& \text { B } 3,0 \text { X } 0+B 3,1 X 1+\ldots+\text { B } 3, n-1 X n-1=S 1 \\
& \text { B n }-1,0 \text { X } 0+B n-1,1 X 1+\ldots \ldots+B n-1, n-1 X n-1=S n-1
\end{aligned}
$$

Where $\mathrm{X} \mathrm{j}$ are the values to be found and they are unknown, Bi,j and $\mathrm{Si}$ are values which are constant,

A practical variant of the problem requires solutions of several linear systems with the same matrix A on the lefthand side. That is, the problem is to find $\mathrm{B}$ matrix $\mathrm{X}=(\mathrm{xi}, \mathrm{x} 2, \ldots, \mathrm{x} \mathrm{m})$ such that

$\mathrm{BX}=\mathrm{S}$

Where $\mathrm{S}=(\mathrm{S} 1, \mathrm{~S} 2 \ldots \ldots, \mathrm{Sm})$ is an $\mathrm{n} \mathrm{x}$ matrix.

There are many methods for solving the system of linear equations $\mathrm{Bx}=\mathrm{S}$. Different methods might require different amounts of work. With a single processor, the complicate time for such problem require O (n3) time of arithmetic operations. The total number of arithmetic operations performed remains the same if using single processor only.

In the system of linear equations that is represented by $\mathrm{Bx}=\mathrm{S}$, where $\mathrm{B}$ is an $\mathrm{N} \times \mathrm{N}$ nonsingular coefficient matrix, $\mathrm{x}$ is an $\mathrm{N} \times 1$ unknowns vector and $\mathrm{S}$ is an $\mathrm{N} \times 1$ known right-hand side vector. When there are multiple right-hand sides, the unknowns are computed for each right-hand side vector one-by-one. According to the solution method applied, the type of the coefficient matrix A, may vary as follows: Dense or sparse, Symmetric or unsymmetric, Positive definite or non-singular.

There are different solution methods which work more efficiently depending on the nature of the coefficient matrix A. These methods can be classified into the following two groups although there are methods that utilize the features of both methods:

Direct methods: These methods give the exact solution of a linear system with known number of operations. There are mainly two different approaches in direct solution methods: (1) finding the inverse of the coefficient matrix and multiplying it with the right hand side vector or (2) transforming the coefficient matrix into triangular or diagonal form in order to decrease the coupling between the equations. The first method is seldom used due to the large number of operations. The most commonly used transformation based direct methods are Gauss elimination.

However, if we use parallel system then the total time will be reduced as a result of sharing the work among the processors, even though some additional overhead may be introduced by necessary communication or synchronization among the tasks and processors. Thus, when using an algorithm for solving the system of linear equations on parallel computers, it is natural that an algorithm with the least number of arithmetic operations is first chosen among serial algorithms. The chosen algorithm is then restructured for parallel computers according to their architecture. Among the different algorithms Gaussian elimination is the ideal candidate for parallel computers to solve linear systems.

\section{The Gaussian Algorithm for Solving Linear Equation}

For a matrix A the Gaussian Algorithm will modify it by making arithmetic operations and transform the matrix from one state to another without changing the solution and this is done either by addition or multiplication, the resulted transformation will be the same and equivalent for the original matrix which is triangular matrix then the vector of the solution will be gotten directly.

\subsection{Sequential Gaussian Algorithm}

The focus of Gaussian Sequential Algorithm to make different operation to the original matrix to obtain equivalent matrix of the linear equations and these transformation will not affect the solution of the linear equations and that's why they are called equivalent, these transformations are mathematical operations i.e. multiplying any matrix raw of a certain equation by a constant nonzero value, equations permutation, adding one equation to the next equation that exists in the matrix (Dumas, and Villard, 2002).

Te number of steps that is required for solving linear equation system with $\mathrm{N} * \mathrm{~N}$ matrix and a vector $\mathrm{S}$ of $\mathrm{N} x 1$ is N-1 Step, through the iteration of the algorithm and in any $i$ iteration any non zero value lies below the diagonal in column $i$ are changed by replacing with every $j$ row, where as $i+1 \leq j<n$, replaced by the sum of row $j$ and aj, I /ai,i multiplied by row I (Dumas, 2002).

Gaussian elimination partial pivoting:

In Gaussian elimination through iteration i, the pivot row will be the i row, and this row will be used to in changing all of them on zero values to zero that lies below the diagonal column $i$. 
In iteration $i$, rows $i$ up to row $n-1$ are explored and examined for the row whose column $i$ values have the biggest absolute value after that, they found row is changed by swapping (pivoted) with row i. , the Pseudo-code of Gaussian elimination are shown in figure 1.

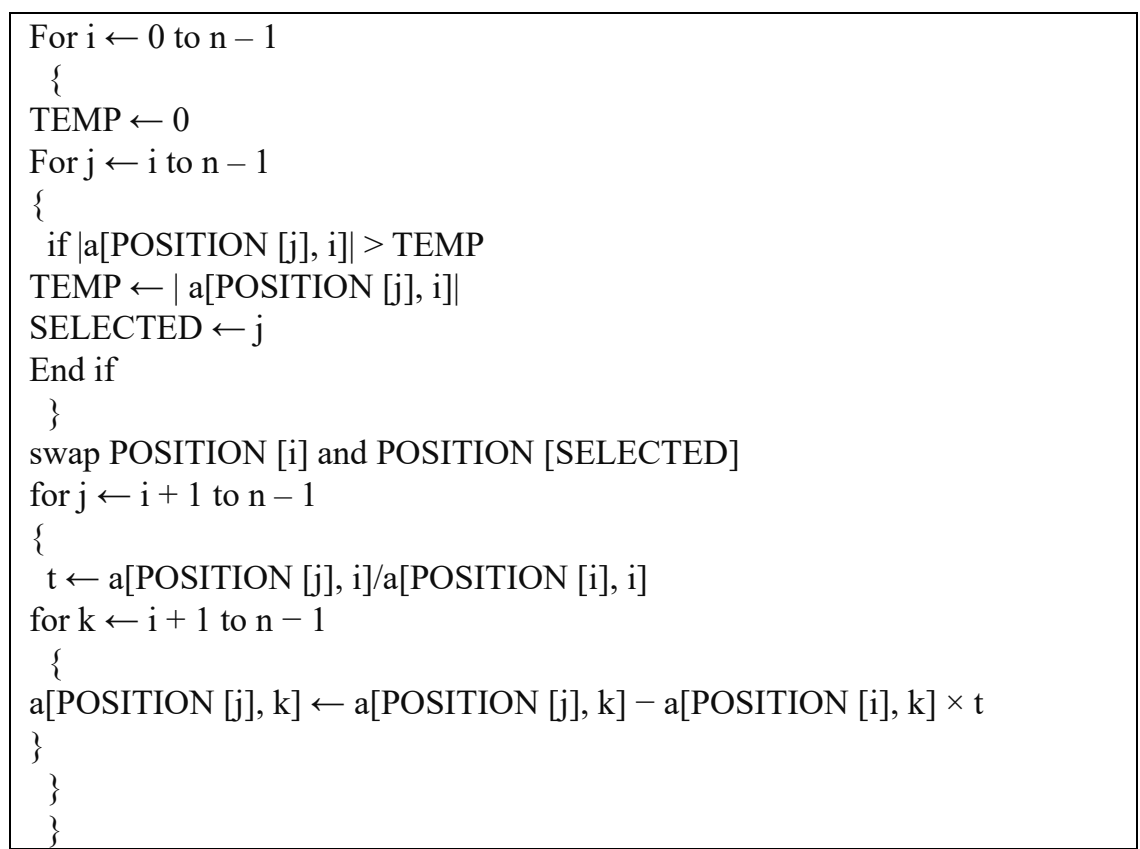

Figure 1. Gaussian elimination Pseudo-code for (pivoting)

\subsection{Parallel Gaussian Elimination}

For testing the parallel performance on super computer we used the Successive Gaussian Elimination (SGE) algorithm for parallel solution of linear equations proposed by MURTHY, 1995. the SGE algorithm does not have a separate back substitution phase, which requires $\mathrm{O}(\mathrm{N})$ steps using $\mathrm{O}(\mathrm{N})$ processors or $\mathrm{O}(\log 2 \mathrm{~N})$ steps using $\mathrm{O}$ (N3) processors, for solving a system of linear algebraic equations. It replaces the back substitution phase by only one step division and possesses numerical stability through partial pivoting as shown in figure 2. Further, in this paper, the SGE algorithm is shown to produce the diagonal form in the same amount of parallel time required for producing triangular form using the conventional parallel GE algorithm. Finally, the effectiveness of the SGE algorithm is demonstrated by studying its performance on a hypercube multiprocessor system.

Solving a linear equation using Gaussian parallel Algorithm is divided into two parts the first part is the Gaussian elimination part; The main aim of Gaussian elimination is to reduce the upper triangle of the matrix that represent linear system to by a steps elimination to give a coefficient matrix.

The estimations of elements are ascertained. The estimation of the variable $\mathrm{x} \mathrm{n-1} \mathrm{might} \mathrm{be} \mathrm{ascertained} \mathrm{from} \mathrm{the}$ last condition of the changed framework. After that it gets to be distinctly conceivable to discover the estimation of the variable $\mathrm{x}$ n-2 from the second to last condition and so on. Illustration of the pivot, Zero elements will not changed and Non Zero elements (variables) will not changed.The Gaussian arranges comprises in successive end of the questions in the conditions of the direct condition framework being comprehended. All the fundamental calculations might be depicted by the accompanying relations.

All the Non Zero elements(variables), which are located lower than the main diagonal and to the left of column i are already zero. At i-th iteration of the Gaussian elimination stage the coefficients of column i located lower than the main diagonal are set to zero. It is done by means of subtracting the row i multiplied by the adequate nonzero constant. After executing (n-1) similar iterations the matrix of linear equation coefficients is transformed in the upper triangle form during the execution of the Gaussian, the matrix element, the pivot will be utilized solving other elements, and the corner to corner component of the turn line is known as the turn component. As it can be noted it is conceivable to perform calculations just if the main component is a nonzero esteem. In addition, if the turn component has a little esteem, then the division and the increase of lines by this component may prompt to aggregation of the computational blunders and the computational insecurity of the calculation. A conceivable approach to maintain a strategic distance from this issue may comprise in the accompanying. At every emphasis of the Gaussian disposal arrange it is important to decide the coefficient with the greatest supreme. 


\section{Parallel Analysis}

Parallel Solving linear equation is analyzed according to the number of communication steps, complexity, speed, and execution time.

Communication steps: this includes the number of steps required for data splitting and results gathering. The number of communication steps that are required to scatter the data depends on the number of processors $\mathrm{P}$, which is $\log \mathrm{p}$. we need same number of communication steps to gather the results from all processors. Complexity: this is the time required to perform calculations locally on each processor.

Speed: this is the communication steps times the speed of the electrical links. Assuming that the speed of electrical links $=250 \mathrm{Mb} / \mathrm{s}$, the speed is $2 \times \log \mathrm{p} \times 250 \mathrm{Mb} / \mathrm{s}$.

Execution time: this is the complexity of matrix splitting, matrix operation and substitution + communication time. The communication time depends on the data that is transmitted for each processor in each step.

\section{Performance Evaluation}

This section represent the Evaluation of the performance for solving the linear equation problem and the matrix multiplication on IMAN1 Zaina cluster supercomputer. The Parallel performance results (run time evaluation, speed up and efficiency) and multi-threaded results will be presented. For the Performance Evaluation purpose we ran different input sizes $(128,256,512,1024,2048,4096,8192)$ using different number of processors $(2,4,8,16,32,64)$. An open MPI library is used in our implementation. The hardware and software (operating system, compiler, MPI) characteristics that are used for implementation are shown in table 4:

Table 4. Hardware and Software characteristics used to for the evaluation

\begin{tabular}{lc}
\hline Hardware & Dual Quad Core Intel Xeon, CPU with SMP, 16 GB RAM \\
\hline Operating system & Scientific Linux 6.4 \\
Compiler, MPI & Open MPI 1.5.4, C Compiler. \\
Matrix Input Size & $(128,256,512,1024,2048,4096,8192)$ Byte \\
Number of Processors & $1,2,4,8,16,32,64$ \\
\hline
\end{tabular}

\subsection{Parallel Run Time Evaluation}

The solving linear equation algorithm has been evaluated according to different data sizes as shown in figure (5). The algorithm has been evaluated with different input matrix sizes $(128 * 128,256 * 256,512 * 512,1024 * 1024$, $2048 * 2048,4096 * 4096,8192 * 8192)$ and number of processors $(2,4,8,16,32,64)$.

The results in figure 5 shows that as the data size increased the run time increased in all processors. This might be due to; first the increased number of matrix elements which increase the complexinty of pivot finding. Second the increased number of splitted elements of the matrix, third the increased time required for gathering the elements and finding the solution of the linear equation. As illustrated from figure 5 we can also observe that the runtime for processor number of 64 was the fastest to solve the linear equation. However, processor number is 2 had the highest run time.

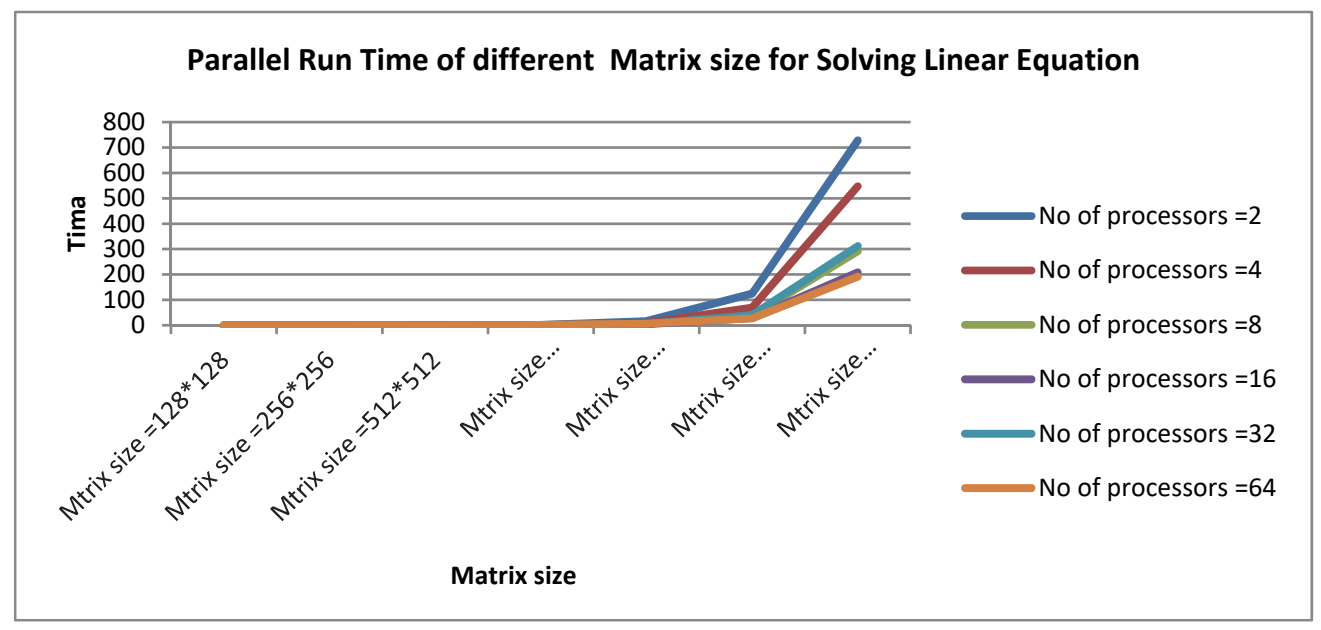


Figure 5. Parallel Run Time of different Matrix size for Solving Linear Equation

Figure 6 and Figure 7 illustrate the run time using different number of processors $(2,4,8,16,32$, and 64). Expiremented by different input data sizes small and large respectively for the small input data size we used $(128,256,512)$ matrix size and for the large input size we used $(1024,2048,4096,8192)$ matrix size.

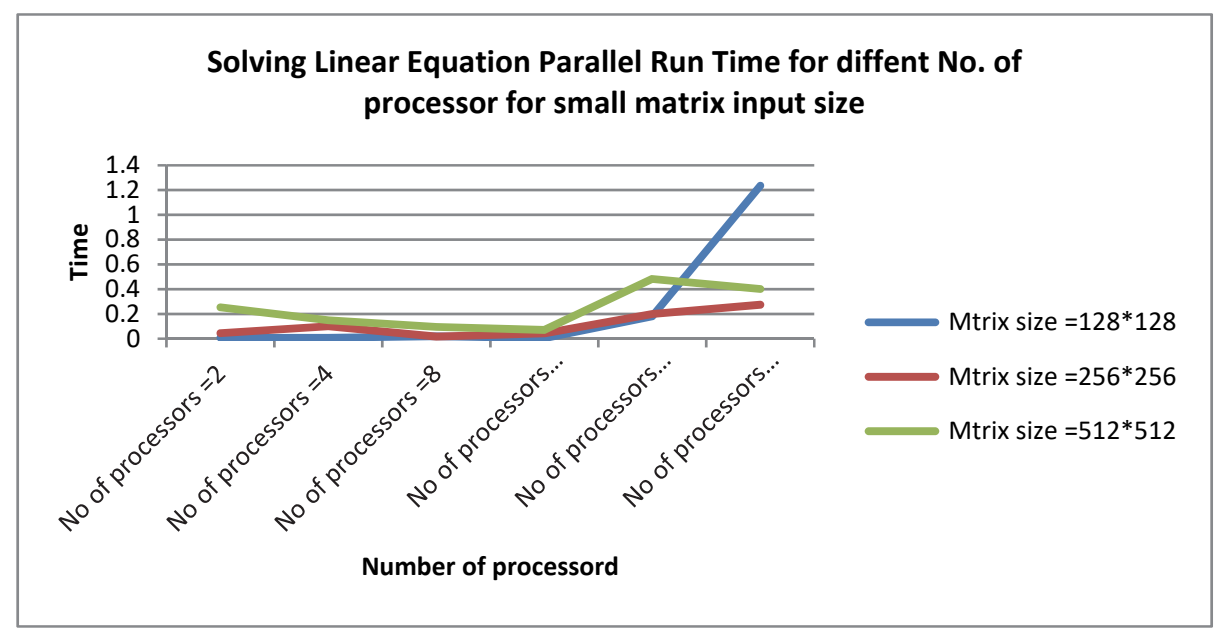

Figure 6. Solving Linear Equation Parallel Run Time for different No. of processor $(2,4,8,16,32,64)$ for small matrix input size

Figure 6 indicates that for the small matrix input size $(128 * 128)$ the running time increases as the number of processors increase. Acordingly running the data on 64 processors resulted in the highest running time although it has the smallest input data size. This can be explained by the fact that the time required for communication between the 64 processors is very high compared to the computation time needed to calculate the small input data size.On the other hand when the number of processors was small (2 processors) the running was the smallest.

Moreover at input data size 512 the running time was the highest over the three matrix sizes ((128*128), (256*256), $(512 * 512))$ using 32 processors. Because as the matrix size increase the running time increases as well for a certain threshold (number of processors, in this case 32 processors) but then it decreases after that threshold, as shown in figure 6 with 64 processors. This might be due to the increased communication overhead between processor which will decrease the benefits of parallelism.

Solving Linear Equation Parallel Run Time for different No. of processor for large matrix input size

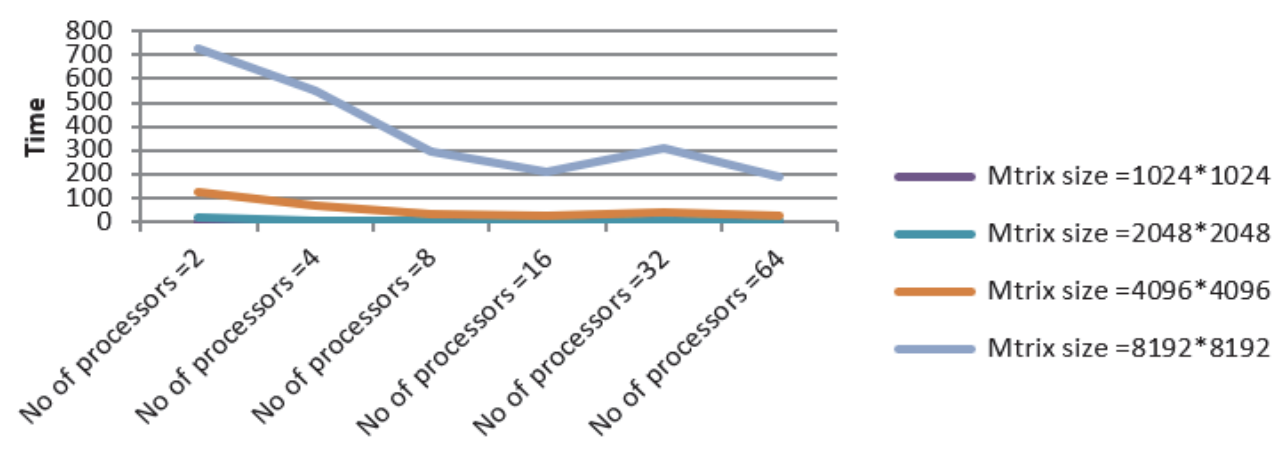

Number of processord

Figure 7. Solving Linear Equation Parallel Run Time for different No. of processor for large matrix input size

For the large matrix input data size $(1024,2048,4096,8192)$, the same settings (number of processors: 2, 4, 8, 16, $32,64)$ as the small input data size was used. Based on figure 7 , it can be noticed that when running the data at 64 
processor the run time for the solving linear equation algorithm for the large matrix size of $8192 * 8192$ decreased as the number of processors increased with the smallest running time. This is because it has the largest size and accordingly, using parallel processing resulted in a reduced running time that is required to solve the linear equation. This reduction in the running time and coputation performance show the power and success of parallel computing in solving linear equations. The overall observation in Figure 7 indicates that there is a decrease in the running time as the number of processors increase; the largest time was at processor 2 and the smallest time at processor 64. As the number of processors increases, the run time is reduced due to better parallelism.

\subsection{Multithreaded Run Time Evaluation}

For the multithreaded running time evaluation in solving linear equation, the algorithm has been evaluated using different input data sizes $(128 * 128,256 * 256,512 * 512,1024 * 1024,2048 * 2048,4096 * 4096,8192 * 8192)$ figure 8 . The algorithm has been also evaluated with different number of threads of $(2,4,8,16,32,64)$. Figure 8 shows that as the data size increases, the run time increases and this is the result of the increased input data size which increases the calculation time that is needed for solving the linear equation since the complexity time of solving linear equation is very high.

The results also show, that the speedup (execution time of one-threaded sequential algorithm compared with parallel multithreaded algorithm) is almost independent of the number of threads. figure 8 also shows a very slow decreases when the number of threads increases. The reasons for this are the heavy thread communication and context switching between multiple threads. However the computation time when running the data at two processors has the lowest time.

Solving Linear Equation multithreaded Run Time for diffent matrix input size

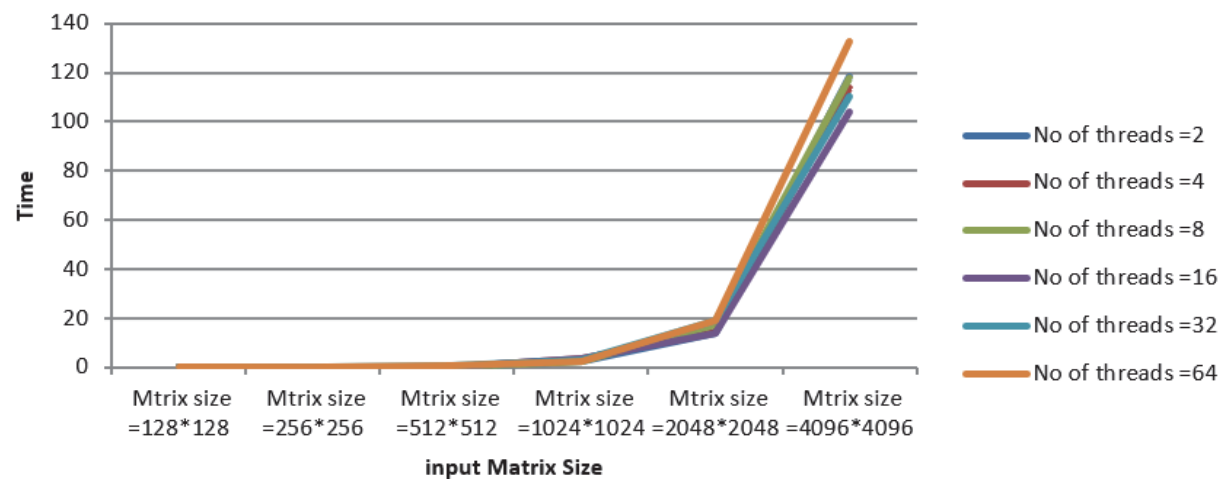

Figure 8. Solving Linear Equation multithreaded Run Time for different matrix input size

\subsection{Sequential Running Time}

For the sequential running time evaluation, solving linear equation algorithm has been evaluated using different input data sizes $(128 * 128,256 * 256,512 * 512,1024 * 1024,2048 * 2048,4096 * 4096,8192 * 8192)$. As illustrated in figure 9 the running time for the Sequential algorithm of solving linear equation increased rapidly with the increase of matrix size.

Solving Linear Equation Sequential Run Time for diffent matrix input size

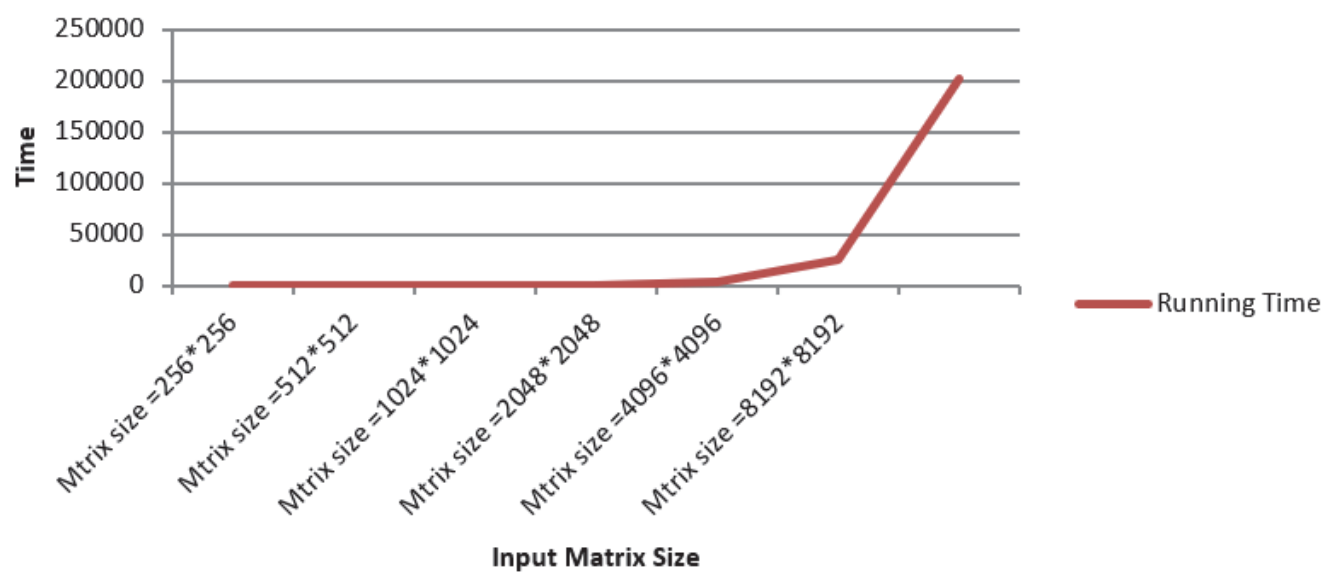


Figure 9. Solving Linear Equation Sequential Run Time for different matrix input size

\subsection{Relative Speedup Evaluation}

The speedup is the ratio between the sequential time and the parallel time (Eq. 1). The relative speedup of the implemented algorithm over the different input matrix size and over the different number of processors as shown in figure 10 .

Relative Speedup $=$ Ts/Tp

Eq. 1

Where: $\mathrm{p}=\#$ of processors;

Ts = execution time of the sequential algorithm;

$\mathrm{Tp}=$ execution time of the parallel algorithm with $\mathrm{p}$ processors.

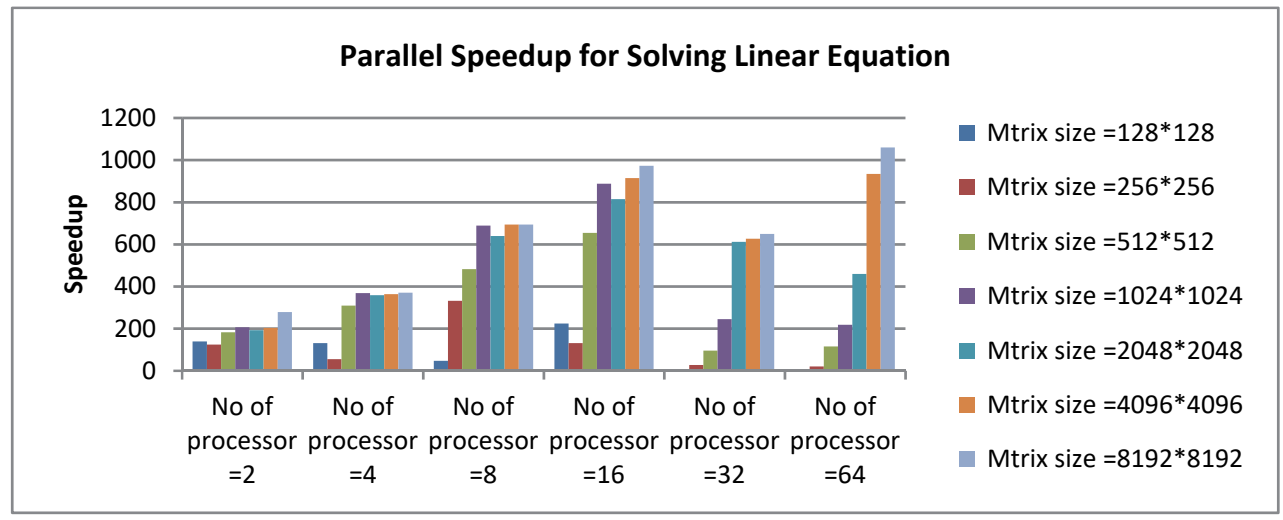

Figure 10. Parallel Speedup for Solving Linear Equation

\subsection{Parallel Efficiency Evaluation}

Parallel efficiency is the ratio between speedup and the number of processors. The parallel efficiency for the algorithm has been evaluated with different matrix size (Eq. 2). as shown in figure 11.

Efficiency $=$ speedup $/$ number of processors

Eq. 2

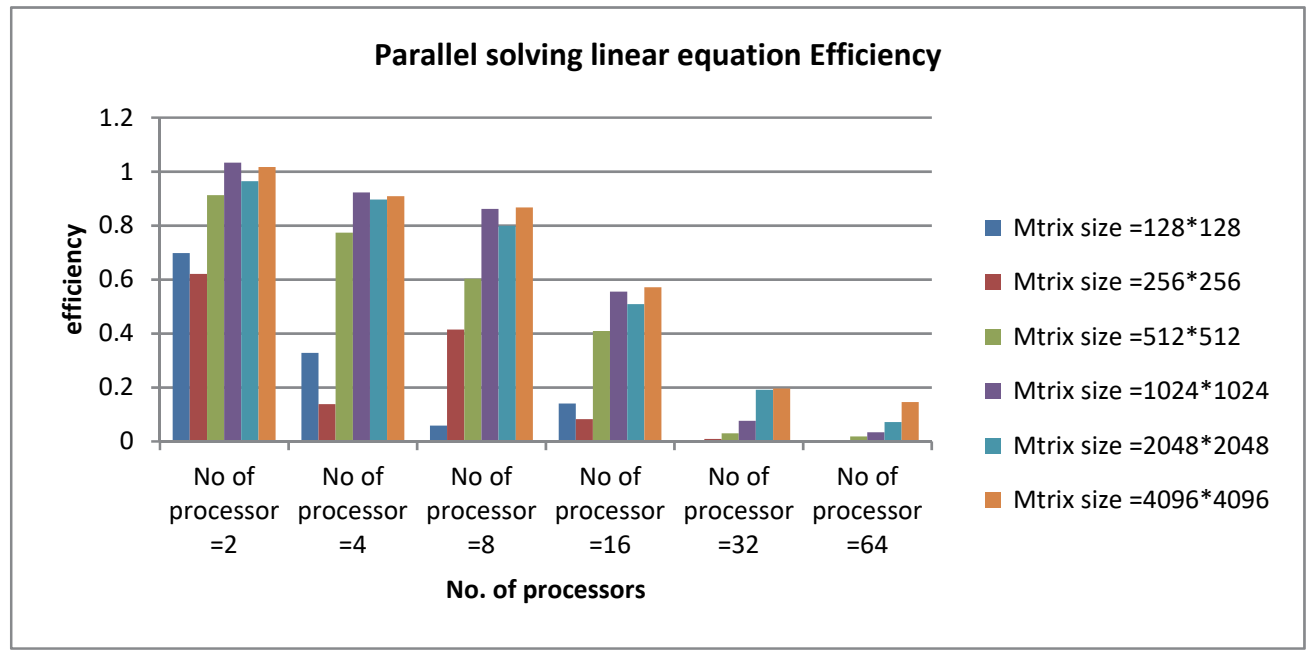

Figure 11. Parallel Efficiency for Solving Linear Equation

\section{Conclusion}

In this paper we evaluated the performance of solving the linear equation application using parallel data processing and MPI over a supercomputer that consist of clusters of 64 processors that run in parallel. Different input 
datasizes has been expiremented with different processors number.For large input data size, the results showed that there is a noticeable decrease in running time as the number of processors increase. The performance of the multithreaded algorithm has been also evaluated. The running time, speedup and efficiency has shown that the performance of the parallel computing over the large matrix size outperformed the sequential and multthereaded methods.

\section{References}

Amjad, A. H., Hussam, N. F., Fatima, E. A. A., \& Sandi, N. F. (2017). A Survey about Self-Healing Systems (Desktop and Web Application), Scientific Research Publishing

Atif, M., \& Rauf, A. (2009, October). Efficient implementation of Gaussian elimination method to recover generator polynomials of convolutional codes. In Emerging Technologies, 2009. ICET 2009. International Conference on (pp. 153-156). IEEE.

Basim, A., Hussam, N. F., \& Omar, S. A. (2006). cDNA microarray genome image processing using fixed spot position. American Journal of Applied Sciences, 3(2), 1730-1734.

Basim, A., \& Hussam, N. F. (2008). Automation of iron difficiency anemia blue and red cell number calculating by intictinal villi tissue slide images enhancing and processing, Computer Science and Information Technology, 2008. ICCSIT'08. International Conference.

Basim, A., Mohammad, H Zu'bi, \& Hussam, N. S. (2007). Mammogram breast cancer image detection using image processing functions. Information Technology Journal, 6(2), 217-221.

Delić, S., \& Jurić, Ž. (2013, May). Some improvements of the gaussian elimination method for solving simultaneous linear equations. In Information \& Communication Technology Electronics \& Microelectronics (MIPRO), 2013 36th International Convention on (pp. 96-101). IEEE.

Dumas, J. G., \& Villard, G. (2001). Computing the rank of large sparse matrices over finite fields. In: CASC'2002 Computer Algebra in Scientific Computing. pp. 22-27. Springer-Verlag.

Dumas, J. G., Gautier, T., Giesbrecht, M., Giorgi, P., Hovinen, B., Kaltofen, E., Saunders, B. D., Turner, W. J., \& Villard, G. (2001). LinBox: A Generic Library for ExactLinear Algebra. In: Cohen, A., Gao, X.S., Takayama, N. (eds.) Mathematical Software: ICMS 2002 (Proceedings of the first International Congress of Mathematical Software. pp. 40-50. World Scientific (2002).

Iyengar, S. R. K., \& Jain, R. K. (2006). Mathematical Methods, Narosa Publishing House Private. Limited. Retrieved March 1, 2017, from http://www.iman1.jo/iman1/

Kaltofen, E., \& Pan, V. (1991, June). Processor efficient parallel solution of linear systems over an abstract field. In Proceedings of the third annual ACM symposium on Parallel algorithms and architectures (pp. 180-191). ACM.

Maria, S., Sheza, N., Sundas, R., Rabea, M., \& Rabiya, I. G. (2015). Elimination Method-A Study of Applications Global Journal of Science Frontier Research: F Mathematics and Decision Sciences, 15(5). Version 1.0 Year 2015 Type: Double Blind Peer Reviewed International Research Journal Publisher: Global Journals Inc. (USA) Online ISSN: 2249-4626 \& Print ISSN: 0975-5896

Mohammad, Q., \& Khattab, H. (2015). New Routing Algorithm for Hex-Cell Network. International Journal of Future Generation Communication and Networking, 8(2), 295-306.

Murthy, K. B., \& Murthy, C. S. R. (1994, August). A new Gaussian elimination-based algorithm for parallel solution of linear equations. In TENCON'94. IEEE Region 10's Ninth Annual International Conference. Theme: Frontiers of Computer Technology. Proceedings of 1994 (pp. 82-85). IEEE.

Pasetto, D., \& Akhriev, A. (2011, October). A comparative study of parallel sort algorithms. In Proceedings of the $\mathrm{ACM}$ international conference companion on Object oriented programming systems languages and applications companion (pp. 203-204). ACM.

Rajalakshmi, K. (2009). Parallel algorithm for solving large system of simultaneous linear equations. International Journal of Computer Science and Network Security, 9(7), 276-279.

Rajalakshmi, K. (2009). Proposed a Parallel Algorithm for Solving Large System of Simultaneous Linear Equations

Rizik MH Al-Sayyed, Hussam N Fakhouri, Ali Rodan, Colin Pattinson, 2017 Polar Particle Swarm Algorithm for Solving Cloud Data Migration Optimization Problem. Modern Applied Science, 11(8), 98. 
Scholl, S., Stumm, C., \& Wehn, N. (2013, September). Hardware implementations of Gaussian elimination over GF (2) for channel decoding algorithms. In AFRICON, 2013 (pp. 1-5). IEEE.

\section{Copyrights}

Copyright for this article is retained by the author(s), with first publication rights granted to the journal.

This is an open-access article distributed under the terms and conditions of the Creative Commons Attribution license (http://creativecommons.org/licenses/by/4.0/). 